\title{
On the growth factor upper bound for Aasen's algorithm *
}

\author{
Yuehua Feng ${ }^{\dagger} \quad$ Linzhang $\mathrm{Lu}^{\ddagger}$
}

August 23, 2018

\begin{abstract}
Aasen's algorithm factorizes a symmetric indefinite matrix $A$ as $A=P^{T} L T L^{T} P$, where $P$ is a permutation matrix, $L$ is unit lower triangular with its first column being the first column of the identity matrix, and $T$ is tridiagonal. In this note, we provide a growth factor upper bound for Aasen's algorithm which is much smaller than that given by Higham. We also show that the upper bound we have given is not tight when the matrix dimension is greater than or equal to 6 .
\end{abstract}

Keywords: Aasen's algorithm, growth factor, $L T L^{T}$ factorization

AMS subject classifications. 15A23, 65F05, 65G50

\section{Introduction}

Aasen's algorithm [1] factorizes a symmetric indefinite matrix $A \in \mathbb{R}^{n \times n}$ as

$$
P A P^{T}=L T L^{T}
$$

where $L$ is unit lower triangular with first column $e_{1}$ (the first column of the identity matrix), $T$ is symmetric tridiagonal, and $P$ is a permutation matrix chosen such that $\left|l_{i j}\right| \leqslant 1$. As it is well known that we can effectively solve symmetric indefinite linear systems $A x=b$ when $A$ is a dense matrix, Aasen's algorithm has already been incorporated into LAPACK [2].

For Aasen's algorithm, Higham gave a growth factor upper bound, which is $4^{n-2}$. However, whether this bound is attainable for $n \geqslant 4$ is an open problem [3]. Cheng [4] constructed an example to obtain the growth factor of 4 for $n=3$, and reported experiments using direct search method in which he obtained growth of 7.99 for $n=4$ and 14.61 for $n=5$.

In this note, we report that the growth factor upper bound for Aasen's algorithm is $2^{n-1}$, which is much smaller than $4^{n-2}$ given by Higham. Moreover, we also show that the upper bound $2^{n-1}$ we have obtained is not tight when $n \geqslant 6$.

${ }^{*}$ This work was supported in part by National Natural Science Foundation of China grant 11671105 and the CSC (grant 201606310121).

${ }^{\dagger}$ School of Mathematical Science, Xiamen University, China. E-mail: yhfeng@stu.xmu.edu.cn.

${ }^{\ddagger}$ School of Mathematical Science, Guizhou Normal University \& School of Mathematical Science, Xiamen University, China. E-mail: llz@gznu.edu.cn,lzlu@xmu.edu.cn. 
This paper is organized as follows. Section 2 presents the upper bounds on entries of the tridiagonal matrix $T$ in Aasen's algorithm. Section 3 reports the growth factor bound for Aasen's algorithm. We draw some conclusions in Section 4. Without loss of generality, we assume that $\max _{i, j}\left|a_{i j}\right|=1$ for the symmetric matrix $A=\left(a_{i j}\right)_{i, j=1}^{n} \in \mathbb{R}^{n \times n}$ and $A \stackrel{\text { def }}{=} P A P^{T}$ for convenience.

\section{Upper bounds on entries of $T$}

We first present a lemma which gives upper bounds on entries of the factor matrix $T$.

Lemma 2.1. Let $A=\left(a_{i j}\right)_{i, j=1}^{n} \in \mathbb{R}^{n \times n}$ be symmetric, $\max _{i, j}\left|a_{i j}\right|=1$ and suppose Aasen's algorithm produces

$$
A \stackrel{\text { def }}{=} P A P^{T}=L T L^{T}
$$

where $L=\left(l_{i j}\right)_{i, j=1}^{n}$ is an unit lower triangular matrix with first column $e_{1},\left|l_{i j}\right| \leqslant 1$ and $T=\left(t_{i j}\right)_{i, j=1}^{n}$ is a symmetric tridiagonal matrix. Then the following inequalities hold:

$$
\left\{\begin{array}{l}
\left|t_{11}\right| \leqslant 1, \quad\left|t_{21}\right| \leqslant 1, \quad\left|t_{22}\right| \leqslant 1, \quad\left|l_{i 2} t_{21}\right| \leqslant 1, \quad 3 \leqslant i \leqslant n, \\
\left|l_{i, j-1} t_{j-1, j}+l_{i j} t_{j j}+l_{i, j+1} t_{j+1, j}\right| \leqslant 2^{j-2}, \quad 2 \leqslant j<i \leqslant n, \\
\left|l_{n, n-1} t_{n-1, n}+t_{n n}\right| \leqslant 2^{n-2}, \\
\left|t_{i, i-1}\right| \leqslant 2^{i-2}, \quad\left|t_{i i}\right| \leqslant 2^{i-1}, \quad 3 \leqslant i \leqslant n .
\end{array}\right.
$$

Proof. We proceed by induction on $n$. If $n=3$, the result is trivial (see the example in Cheng [4]). Assume that it holds true for $n=k$. Consider $n=k+1$. By direct calculation and $2 \leqslant q \leqslant k$,

$$
\left\{\begin{array}{l}
a_{k+1,1}=l_{k+1,2} t_{21} \\
a_{k+1, q}=\sum_{j=2}^{q} l_{q j}\left(l_{k+1, j-1} t_{j-1, j}+l_{k+1, j} t_{j j}+l_{k+1, j+1} t_{j+1, j}\right), \\
a_{k+1, k+1}=\sum_{j=2}^{k} l_{k+1, j}\left(l_{k+1, j-1} t_{j-1, j}+l_{k+1, j} t_{j j}+l_{k+1, j+1} t_{j+1, j}\right) \\
+l_{k+1, k} t_{k, k+1}+t_{k+1, k+1} .
\end{array}\right.
$$

Since $\max _{i, j}\left|a_{i j}\right|=1$, then

$$
\left|l_{k+1,2} t_{21}\right| \leqslant 1
$$

By (3b) and $\left|l_{g h}\right| \leqslant 1(1 \leqslant g, h \leqslant n)$, then

$$
\begin{gathered}
\left|l_{k+1,2} t_{22}+l_{k+1,3} t_{32}\right| \leqslant 1 \\
\left|l_{k+1,2} t_{23}+l_{k+1,3} t_{33}+l_{k+1,4} t_{43}\right| \leqslant 2 \\
\left|l_{k+1,3} t_{34}+l_{k+1,4} t_{44}+l_{k+1,5} t_{54}\right| \leqslant 2^{2} \\
\vdots \\
\left|l_{k+1, k-2} t_{k-2, k-1}+l_{k+1, k-1} t_{k-1, k-1}+l_{k+1, k} t_{k, k-1}\right| \leqslant 2^{k-3}
\end{gathered}
$$




$$
\left|l_{k+1, k-1} t_{k-1, k}+l_{k+1, k} t_{k k}+l_{k+1, k+1} t_{k+1, k}\right| \leqslant 2^{k-2} .
$$

Furthermore, from (3c)

$$
\left|l_{k+1, k} t_{k, k+1}+t_{k+1, k+1}\right| \leqslant \sum_{j=2}^{k} 2^{j-2}+1=2^{k-1} .
$$

Rewrite (3b) as

$$
\begin{aligned}
a_{k+1, k} & =\sum_{j=2}^{k} l_{k j}\left(l_{k+1, j-1} t_{j-1, j}+l_{k+1, j} t_{j j}+l_{k+1, j+1} t_{j+1, j}\right) \\
& =\sum_{j=2}^{k-1} l_{k+1, j}\left(l_{k, j-1} t_{j, j-1}+l_{k j} t_{j j}+l_{k, j+1} t_{j, j+1}\right) \\
& +l_{k+1, k}\left(l_{k, k-1} t_{k, k-1}+t_{k k}\right)+t_{k+1, k} .
\end{aligned}
$$

Apply the induction hypothesis,

$$
\left|t_{k+1, k}\right| \leqslant \sum_{j=2}^{k-1} 2^{j-2}+2^{k-2}+1=2^{k-1},
$$

by (4),

$$
\left|t_{k+1, k+1}\right| \leqslant 2^{k}
$$

Therefore, when $n=k+1$ the lemma still holds true. We thus prove the lemma.

\section{Growth factor bound for Aasen's algorithm}

In this section, we present our main result. From Lemma 2.1 and if $\left|t_{n, n}\right|=2^{n-1}$, without loss of generality, we assume that $t_{n, n}=2^{n-1}$, then by (3c) with $k=n-1$,

$$
\left\{\begin{array}{l}
l_{n j}\left(l_{n, j-1} t_{j-1, j}+l_{n j} t_{j j}+l_{n, j+1} t_{j+1, j}\right)=-2^{j-2}, \quad 2 \leqslant j \leqslant n-1, \\
l_{n, n-1} t_{n-1, n}=-2^{n-2} \\
t_{n n}=2^{n-1}
\end{array}\right.
$$

By (5) and (2b) - (2d),

$$
l_{n j}= \pm 1, \quad 2 \leqslant j \leqslant n-1 .
$$

Substituting (5) and (6) into (3b) with $k=n-1$, we obtain

$$
\left|\sum_{j=2}^{q} l_{q j} l_{n j} 2^{j-2}\right| \leqslant 1, \quad 2 \leqslant q \leqslant n-1,
$$

thus

$$
l_{q j} l_{n j} l_{n q}=-1, \quad 2 \leqslant j<q \leqslant n-1,
$$


furthermore,

$$
l_{q j} l_{i j} l_{i q}=-1, \quad 2 \leqslant j<q<i \leqslant n-1 .
$$

Next we will derive an upper bound on $t_{n n}$ from (7) and (8).

At first, we rewrite (5) as

$$
\left\{\begin{array}{l}
l_{n j}\left(l_{n, j-1} t_{j-1, j}+l_{n j} t_{j j}+l_{n, j+1} t_{j+1, j}\right)=-2^{j-2}+\delta_{j-2}, \quad 2 \leqslant j \leqslant n-1, \\
l_{n, n-1} t_{n-1, n}=-2^{n-2}+\delta_{n-2}, \\
t_{n n}=2^{n-1}-\delta
\end{array}\right.
$$

where $\delta=\sum_{j=0}^{n-2} \delta_{j}$ and $0 \leqslant \delta_{j} \leqslant 2^{j+1}(0 \leqslant j \leqslant n-2)$. Hence the upper bound on $t_{n n}$ is equivalent to the following optimization problem

$$
\left\{\begin{array}{l}
\delta=\min \sum_{j=0}^{n-2} \delta_{j} \\
\text { s. t. } \quad 0 \leqslant \delta_{j} \leqslant 2^{j+1}, \quad 0 \leqslant j \leqslant n-2 .
\end{array}\right.
$$

In the following analysis, we will seek more constraints on $\delta_{j}$. Substituting (7) and (9) into (3b),

$$
0 \leqslant \delta_{q-2}-\sum_{j=2}^{q-1} \delta_{j-2} \leqslant 2, \quad 3 \leqslant q \leqslant n-1 .
$$

From (77) and (99),

$$
\left\{\begin{array}{l}
-l_{j, j-1} t_{j-1, j}+t_{j j}-l_{j+1, j} t_{j+1, j}=-2^{j-2}+\delta_{j-2}, \quad 2 \leqslant j \leqslant n-2, \\
-l_{n-1, n-2} t_{n-2, n-1}+t_{n-1, n-1}=2^{n-3}+\delta_{n-3}-\delta_{n-2} \\
l_{n, n-1} t_{n-1, n}=-2^{n-2}+\delta_{n-2} \\
t_{n n}=2^{n-1}-\delta
\end{array}\right.
$$

For $2 \leqslant q<i \leqslant n-1$, multiply $l_{i q}$ on both sides of (3b) with $k=i-1$, then from (8) and (12),

$$
\begin{aligned}
l_{i q} a_{i q}= & \sum_{j=2}^{q} l_{i q} l_{q j}\left(l_{i, j-1} t_{j-1, j}+l_{i j} t_{j j}+l_{i, j+1} t_{j+1, j}\right) \\
& =\left\{\begin{array}{lc}
\sum_{j=2}^{q-1}\left(2^{j-2}-\delta_{j-2}\right)-2^{q-2}+\delta_{q-2}, & q<i-1 \\
\sum_{j=2}^{q-1}\left(2^{j-2}-\delta_{j-2}\right)-2^{q-2}+\delta_{q-2}+2 l_{q+1, q} t_{q+1, q}, & q=i-1
\end{array}\right. \\
& =\left\{\begin{array}{lc}
-1+\delta_{q-2}-\sum_{j=2}^{q-1} \delta_{j-2}, & 2 \leqslant q<i-1 \leqslant n-2, \\
-1+\delta_{q-2}-\sum_{j=2}^{q-1} \delta_{j-2}+2 l_{q+1, q} t_{q+1, q}, & 2 \leqslant q=i-1 \leqslant n-2 .
\end{array}\right.
\end{aligned}
$$


Since $l_{i q}= \pm 1$ and $\left|a_{i q}\right| \leqslant 1$, then $\left|-1+\delta_{q-2}-\sum_{j=2}^{q-1} \delta_{j-2}\right| \leqslant 1$ produces the same condition as (11). For the last equality of (13), we have

$$
-\frac{\delta_{q-2}-\sum_{j=0}^{q-3} \delta_{j}}{2} \leqslant l_{q+1, q} t_{q+1, q} \leqslant 1-\frac{\delta_{q-2}-\sum_{j=0}^{q-3} \delta_{j}}{2}, \quad 2 \leqslant q \leqslant n-2 .
$$

By (8), (12) and $k=i-1$, then (3c) satisfies

$$
a_{i i}=\left\{\begin{array}{lr}
4 l_{i, i-1} t_{i, i-1}+l_{i+1, i} t_{i+1, i}-\sum_{j=0}^{i-2}\left(2^{j}-\delta_{j}\right), & 3 \leqslant i \leqslant n-2, \\
4 l_{i, i-1} t_{i, i-1}+\sum_{j=0}^{i-2} \delta_{j}-\delta_{i-1}+1, & i=n-1 .
\end{array}\right.
$$

Since $\left|a_{i i}\right| \leqslant 1$, then

$$
2^{q-1}-2-\sum_{j=0}^{q-2} \delta_{j} \leqslant 4 l_{q, q-1} t_{q, q-1}+l_{q+1, q} t_{q+1, q} \leqslant 2^{q-1}-\sum_{j=0}^{q-2} \delta_{j}, \quad 3 \leqslant q \leqslant n-2,
$$

and

$$
-\frac{1}{2}+\frac{\delta_{n-2}-\sum_{j=0}^{n-3} \delta_{j}}{4} \leqslant l_{n-1, n-2} t_{n-1, n-2} \leqslant \frac{\delta_{n-2}-\sum_{j=0}^{n-3} \delta_{j}}{4} .
$$

For $3 \leqslant q \leqslant n-2$, from (14) and (16),

$$
\frac{2^{q}-6-3 \sum_{j=0}^{q-3} \delta_{j}-\delta_{q-2}}{8} \leqslant l_{q, q-1} t_{q, q-1} \leqslant \frac{2^{q}-3 \sum_{j=0}^{q-3} \delta_{j}-\delta_{q-2}}{8} .
$$

So (14) and (18) are established simultaneously for $3 \leqslant q \leqslant n-2$ when

$$
2^{q}-14 \leqslant 7 \sum_{j=0}^{q-4} \delta_{j}-\delta_{q-3}+\delta_{q-2} \leqslant 2^{q}, \quad 3 \leqslant q \leqslant n-2 .
$$

Let $q=n-2$ in (14) and from (17),

$$
\left\{\begin{array}{l}
-\frac{\delta_{n-4}-\sum_{j=0}^{n-5} \delta_{j}}{2} \leqslant \frac{\delta_{n-2}-\sum_{j=0}^{n-3} \delta_{j}}{4} \\
-\frac{1}{2}+\frac{\delta_{n-2}-\sum_{j=0}^{n-3} \delta_{j}}{4} \leqslant 1-\frac{\delta_{n-4}-\sum_{j=0}^{n-5} \delta_{j}}{2} .
\end{array}\right.
$$

Hence,

$$
-6 \leqslant 3 \sum_{j=0}^{n-5} \delta_{j}-\delta_{n-4}+\delta_{n-3}-\delta_{n-2} \leqslant 0
$$


Therefore plugging (11), (19) and (20) into the optimization problem (10),

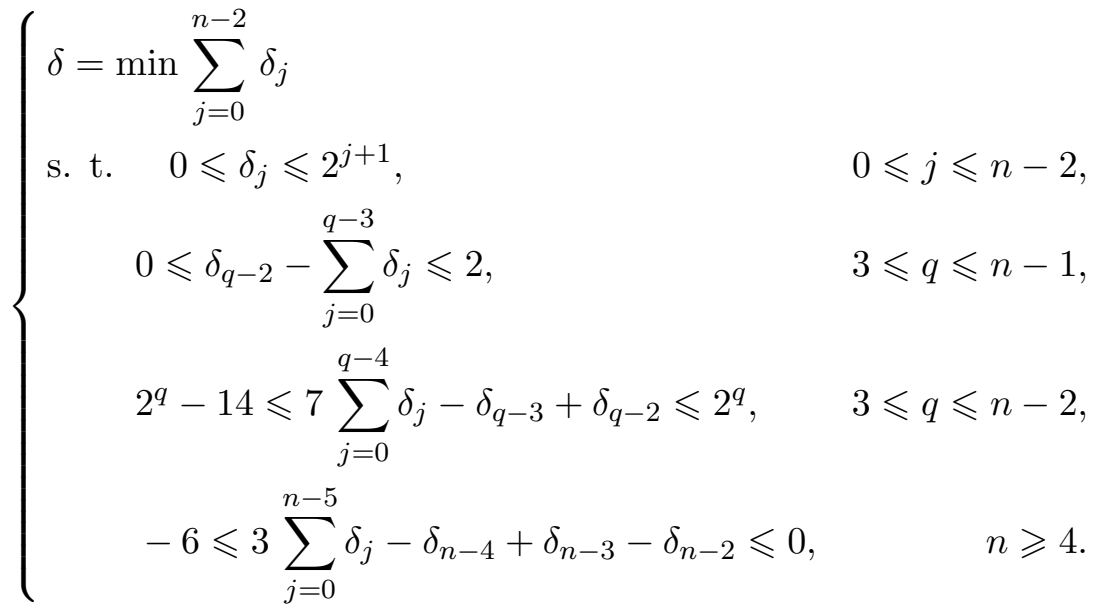

Since $2^{q}-14>0(q \geqslant 4)$ and from (21) , it is easy to obtain

$$
\begin{cases}\delta=0, & n \leqslant 5, \\ \delta>0, & n \geqslant 6 .\end{cases}
$$

Therefore, by (9), $t_{n n}<2^{n-1}$ when $n \geqslant 6$. A similar argument shows that $t_{n n}>-2^{n-1}$ when $n \geqslant 6$. Hence, $\left|t_{n n}\right|<2^{n-1}$ when $n \geqslant 6$, this motivates the following Theorem.

Theorem 3.1. Let a symmetric indefinite matrix $A=\left(a_{i j}\right) \in \mathbb{R}^{n \times n}$, then the growth factor bound for Aasen's algorithm is $2^{n-1}$, but that is not tight when $n \geqslant 6$.

We construct examples with $n=4$ and $n=5$ such that the upper bounds are attainable.

$$
\begin{aligned}
A & =\left(\begin{array}{cccc}
1 & 1 & -1 & 1 \\
1 & \frac{\delta}{2}-1 & 1 & \delta-1 \\
-1 & 1 & 1 & -1 \\
1 & \delta-1 & -1 & 1
\end{array}\right) \\
& =\left(\begin{array}{cccc}
1 & &
\end{array}\right)\left(\begin{array}{cccc}
1 & 1 & & \\
0 & 1 & & \\
0 & -1 & 1 & \\
0 & 1 & 1 & 1+\frac{\delta}{2}
\end{array}\right)\left(\begin{array}{cccc}
\frac{\delta}{2} & \\
& \frac{\delta}{2} & 2+\frac{\delta}{2} & -4 \\
& & -4 & 8-2 \delta
\end{array}\right)\left(\begin{array}{cccc}
1 & 0 & 0 & 0 \\
& 1 & -1 & 1 \\
& & 1 & 1 \\
& & & 1
\end{array}\right),
\end{aligned}
$$

and

$$
\begin{aligned}
A & =\left(\begin{array}{ccccc}
1 & 1 & 1 & 1 & -1 \\
1 & \frac{\delta}{4} & 1-\frac{\delta}{2} & \delta-1 & 1-\delta \\
1 & 1-\frac{\delta}{2} & 1 & 1 & 2 \delta-1 \\
1 & \delta-1 & 1 & 1 & -1 \\
-1 & 1-\delta & 2 \delta-1 & -1 & 1
\end{array}\right) \\
& =\left(\begin{array}{cccccc}
1 & & & \\
0 & 1 & & \\
0 & 1 & 1 & \\
0 & 1 & -1 & 1 \\
0 & -1 & 1 & 1 & 1
\end{array}\right)\left(\begin{array}{ccccccc}
1 & 1 & & & \\
1 & \frac{\delta}{4} & 1 & & \\
& 1-\frac{3 \delta}{4} & -1+\frac{5 \delta}{4} & \delta & & \\
& & \delta & -8 & -8+4 \delta & 16-12 \delta
\end{array}\right)\left(\begin{array}{ccccc}
1 & 0 & 0 & 0 & 0 \\
& 1 & 1 & 1 & -1 \\
& 1 & -1 & 1 \\
& & & 1 & 1 \\
& & & &
\end{array}\right) .
\end{aligned}
$$


When $\delta \rightarrow 0^{+}$in these two matrices $A$, the upper bounds are attainable. For the following $n=6$ example,

$$
\begin{aligned}
& A=\left(\begin{array}{cccccc}
1 & 1 & 1 & 1 & 1 & -1 \\
1 & \delta / 2-3 / 4 & -1 / 2 & \delta-1 & \delta-1 & 1-\delta \\
1 & -1 / 2 & -1 & -1 & 1 & -1 \\
1 & \delta-1 & -1 & 5 \delta-3 & 1 & 2 \delta-1 \\
1 & \delta-1 & 1 & 1 & 1 & -1 \\
-1 & 1-\delta & -1 & 2 \delta-1 & -1 & 1
\end{array}\right) \\
& =\left(\begin{array}{cccccccc}
1 & & & & \\
0 & 1 & & & & \\
0 & 1 & 1 & & & \\
0 & 1 & -1 & 1 & & \\
0 & 1 & -1 & -1 & 1 & \\
0 & -1 & 1 & 1 & 1 & 1
\end{array}\right)\left(\begin{array}{cccccc}
1 & 1 & & & & \\
1 & -\frac{3}{4}+\frac{1}{2} \delta & \frac{1}{4}-\frac{1}{2} \delta & & & \\
& \frac{1}{4}-\frac{1}{2} \delta & -\frac{3}{4}+\frac{1}{2} \delta & -1 & & \\
& & -1 & -3+3 \delta & \delta & \\
& & \delta & 8-3 \delta & -16+8 \delta \\
& & & -16+8 \delta & 32-20 \delta
\end{array}\right) \\
& \left(\begin{array}{cccccc}
1 & & & & \\
0 & 1 & & & \\
0 & 1 & 1 & & & \\
0 & 1 & -1 & 1 & & \\
0 & 1 & -1 & -1 & 1 & \\
0 & -1 & 1 & 1 & 1 & 1
\end{array}\right)^{T}
\end{aligned}
$$

Since $\left|a_{i j}\right| \leq 1$, then $\frac{2}{5} \leq \delta \leq \frac{4}{5}$ and the growth factor is $24<2^{5}$ when $n=6$.

\section{Conclusion}

Variants of Aasen's algorithm have been incorporated into LAPACK, as they can be effectively applied to solve symmetric indefinite linear systems. We estimate the growth factor upper bound for Aasen's algorithm by direct computation and obtain the value $2^{n-1}$, which is much smaller than that given by Higham. We also prove that the bound is not tight when the matrix size is greater than 6 . Besides, some matrix examples are constructed to verify our theoretical analysis with $n=4,5,6$.

\section{References}

[1] Jan Ole Aasen. On the reduction of a symmetric matrix to tridiagonal form. BIT Numerical Mathematics, 11(3):233-242, 1971.

[2] Edward Anderson, Zhaojun Bai, Christian Bischof, L Susan Blackford, James Demmel, Jack Dongarra, Jeremy Du Croz, Anne Greenbaum, Sven Hammarling, Alan McKenney, et al. LAPACK Users' guide. SIAM, 1999.

[3] Nicholas J Higham. Accuracy and stability of numerical algorithms. SIAM, 2002. 
[4] Sheung Hun Cheng. Symmetric indefinite matrices: Linear system solvers and modified inertia problems. PhD thesis, University of Manchester, 1998. 